\title{
The E-ELT first light spectrograph HARMONI: capabilities and modes
}

\section{Thatte, Niranjan, Clarke, Fraser, Bryson, Ian, Shnetler, Hermine, Tecza, Matthias, et al.}

\begin{abstract}
Niranjan A. Thatte, Fraser Clarke, lan Bryson, Hermine Shnetler, Matthias Tecza, Thierry Fusco, Roland M. Bacon, Johan Richard, Evencio Mediavilla, Benoît Neichel, Santiago Arribas, Begoña Garcia-Lorenzo, Christopher J. Evans, Alban Remillieux, Kacem El Madi, Jose Miguel Herreros, Dave Melotte, Kieran O'Brien, lan A. Tosh, Joël Vernet, Peter Hammersley, Derek J. Ives, Gert Finger, Ryan Houghton, Dimitra Rigopoulou, James D. Lynn, Jamie R. Allen, Simon D. Zieleniewski, Sarah Kendrew, Vanessa Ferraro-Wood, Arlette Pécontal-Rousset, Johan Kosmalski, Florence Laurent, Magali Loupias, Laure Piqueras, Edgar Renault, Jeremy Blaizot, Eric Daguisé, Jean-Emmanuel Migniau, Aurélien Jarno, Andy Born, Angus M. Gallie, David M. Montgomery, David Henry, Noah Schwartz, William Taylor, Gérard Zins, Luis Fernando Rodríguez-Ramos, Miguel Cagigas, Giuseppina Battaglia, Refael Rebolo López, Elvio Hernández Suárez, José Vicente Gigante-Ripoll, Javier Piqueras López, Montserrat Villa Martin, Carlos Correia, Sandrine Pascal, Leonardo Blanco, Pascal Vola, Benoit Epinat, Celine Peroux, Arthur Vigan, Kjetil Dohlen, Jean-Francois Sauvage, Martin Lee, Alexis Carlotti, Christophe Verinaud, Tim Morris, Richard Myers, Andrew Reeves, Mark Swinbank, Ariadna Calcines, Marrie Larrieu, "The E-ELT first light spectrograph HARMONI: capabilities and modes," Proc. SPIE 9908, Ground-based and Airborne Instrumentation for Astronomy VI, 99081X (9 August 2016); doi: 10.1117/12.2230629
\end{abstract}

Event: SPIE Astronomical Telescopes + Instrumentation, 2016, Edinburgh, United Kingdom 


\title{
The E-ELT first light spectrograph HARMONI: capabilities and modes
}

Niranjan A. Thatte*a ${ }^{*}$, Fraser Clarke ${ }^{\mathrm{a}}$, Ian Bryson ${ }^{\mathrm{h}}$, Hermine Schnetler ${ }^{\mathrm{h}}$, Matthias Tecza ${ }^{\mathrm{a}}$, Thierry Fusco $^{\mathrm{f}, \mathrm{j}}$, Roland M. Bacon ${ }^{\mathrm{c}}$, Johan Richard ${ }^{\mathrm{c}}$, Evencio Mediavilla ${ }^{\mathrm{d}}$, Benoît Neichel ${ }^{\mathrm{j}}$, Santiago Arribas $^{\mathrm{b}}$, Begona García-Lorenzo ${ }^{\mathrm{d}}$, Christopher J. Evans ${ }^{\mathrm{h}}$, Alban Remillieux ${ }^{\mathrm{c}}$, Kacem El Madi ${ }^{\mathrm{j}}$, José Miguel Herreros ${ }^{\mathrm{d}}$, Dave Melotte ${ }^{\mathrm{h}}$, Kieran O’Brien ${ }^{\mathrm{a}}$, Ian A. Tosh ${ }^{\mathrm{k}}$, Joel Vernet ${ }^{\mathrm{g}}$, Peter Hammersley ${ }^{\mathrm{g}}$, Derek J. Ives ${ }^{\mathrm{g}}$, Gert Finger ${ }^{\mathrm{g}}$, Ryan Houghton ${ }^{\mathrm{a}}$, Dimitra Rigopoulou ${ }^{\mathrm{a}}$, James D. Lynn ${ }^{\mathrm{a}}$, Jamie R. Allen $^{\mathrm{a}}$, Simon D. Zieleniewski ${ }^{\mathrm{a}}$, Sarah Kendrew ${ }^{\mathrm{a}}$, Vanessa Ferraro-Wood ${ }^{\mathrm{a}}$, Arlette PécontalRousset $^{\mathrm{c}}$, Johan Kosmalski ${ }^{\mathrm{c}}$, Florence Laurent ${ }^{\mathrm{c}}$, Magali Loupias ${ }^{\mathrm{c}}$, Laure Piqueras ${ }^{\mathrm{c}}$, Edgar Renault ${ }^{\mathrm{c}}$, Jeremy Blaizot $^{\mathrm{c}}$, Eric Daguiséc ${ }^{\mathrm{c}}$, Jean-Emmanuel Migniau ${ }^{\mathrm{c}}$, , Aurélien Jarno ${ }^{\mathrm{c}}$, Andy Born ${ }^{\mathrm{h}}$, Angus M. Gallie $^{\mathrm{h}}$, David M. Montgomery ${ }^{\mathrm{h}}$, David Henry ${ }^{\mathrm{h}}$, Noah Schwartz ${ }^{\mathrm{h}}$, William Taylor ${ }^{\mathrm{h}}$, Gérard Zins ${ }^{1}$, Luis Fernando Rodríguez-Ramos ${ }^{\mathrm{d}}$, Miguel Cagigas ${ }^{\mathrm{d}}$, Giuseppina Battaglia ${ }^{\mathrm{d}}$, Rafael Rebolo López ${ }^{\mathrm{d}}$, Jorgé Sánchez-Capuchino Revuelta ${ }^{\mathrm{d}}$, José Luis Rasilla ${ }^{\mathrm{d}}$, Elvio Hernández-Suárez ${ }^{\mathrm{d}}$, José Vicente Gigante-Ripoll $^{\mathrm{d}}$, Javier Piqueras López ${ }^{\mathrm{b}}$, Montserrat Villa Martin ${ }^{\mathrm{b}}$, Carlos Correia ${ }^{\mathrm{j}}$, Sandrine Pascal ${ }^{\mathrm{j}}$, Leonardo Blanco ${ }^{j}$, Pascal Vola ${ }^{j}$, Benoit Epinat ${ }^{j}$, Celine Peroux $^{j}$, Arthur Vigan ${ }^{j}$, Kjetil Dohlen $^{j}$, JeanFrancois Sauvage ${ }^{j}$, Martin Lee ${ }^{k}$, Alexis Carlotti ${ }^{1}$, Christophe Verinaud ${ }^{1}$, Tim Morris ${ }^{i}$, Richard Myers ${ }^{i}$, Andrew Reeves ${ }^{i}$, Mark Swinbank ${ }^{i}$, Ariadna Calcines ${ }^{i}$, Marrie Larrieu ${ }^{e}$.

${ }^{\mathrm{a}}$ Dept. of Astrophysics, University of Oxford, Keble Road, Oxford, OX1 3RH, U.K.; ${ }^{\mathrm{b}}$ Centro de Astrobiologica (CSIC/INTA),28850 Torrejón de Ardoz, Madrid, Spain; ${ }^{\mathrm{C} C R A L}$, Observatoire de Lyon, 9 Avenue Charles Andre, 69561 Saint Genis Laval, France; ${ }^{\mathrm{d}} \mathrm{IAC}, \mathrm{C} / \mathrm{Via}$ Lactea, s/n, 38205, La Laguna (Tenerife), Spain; ${ }^{~}$ Observatoire Midi-Pyrénées, 14 avenue Edouard Belin, 31400 Toulouse, France; ${ }^{\mathrm{f} O N E R A, ~ B . P .72, ~ 92322, ~ C h a t i l l o n, ~ F r a n c e ; ~}{ }^{\mathrm{g}}$ ESO, Karl-Schwarzschildstrasse 2, 85748, Garching, Germany; ${ }^{\mathrm{h}}$ UKATC, Royal Observatory Edinburgh, Blackford Hill, Edinburgh, EH9 3HJ, U.K.; ' Department of Physics, Durham University, South Road, Durham DH1 3LE, U.K.; ${ }^{\mathrm{j}}$ Laboratoire d'Astrophysique de Marseille, 38, rue Frédéric Joliot-Curie, 13388 Marseille cedex 13, France; ${ }^{k}$ RAL Space, STFC, Rutherford Appleton Laboratory, Didcot OX11 0QX, U.K.; Institut de Planétologie et d'Astrophysique de Grenoble, 414, Rue de la Piscine, 38400 St-Martin d'Hères,

France.

\begin{abstract}
HARMONI is the E-ELT's first light visible and near-infrared integral field spectrograph. It will provide four different spatial scales, ranging from coarse spaxels of $60 \times 30$ mas best suited for seeing limited observations, to 4 mas spaxels that Nyquist sample the diffraction limited point spread function of the E-ELT at near-infrared wavelengths. Each spaxel scale may be combined with eleven spectral settings, that provide a range of spectral resolving powers $(\mathrm{R} \sim 3500,7500$ and 20000) and instantaneous wavelength coverage spanning the $0.5-2.4 \mu \mathrm{m}$ wavelength range of the instrument. In autumn 2015, the HARMONI project started the Preliminary Design Phase, following signature of the contract to design, build, test and commission the instrument, signed between the European Southern Observatory and the UK Science and Technology Facilities Council. Crucially, the contract also includes the preliminary design of the HARMONI Laser Tomographic Adaptive Optics system. The instrument's technical specifications were finalized in the period leading up to contract signature. In this paper, we report on the first activity carried out during preliminary design, defining the baseline architecture for the system, and the trade-off studies leading up to the choice of baseline.
\end{abstract}

Keywords: integral field spectroscopy, adaptive optics, near-infrared spectroscopy, image slicer, ELT, SCAO, LTAO

Ground-based and Airborne Instrumentation for Astronomy VI, edited by Christopher J. Evans, Luc Simard, Hideki Takami Proc. of SPIE Vol. 9908, 99081X · @ 2016 SPIE · CCC code: 0277-786X/16/\$18 · doi: 10.1117/12.2230629 


\section{SCIENTIFIC MOTIVATION}

Integral field spectroscopy provides an extremely efficient way of obtaining spectra of targets within a modestly sized field-of-view (FoV). This is particularly relevant when using adaptive optics (AO) correction at an Extremely Large Telescope (ELT), as the quasi-diffraction limited spatial resolution of the telescope is substantially smaller than both the seeing and the typical level of differential atmospheric refraction. In addition, rapidly varying night sky and thermal backgrounds, both of which can be comparable to the source brightness in the extraction aperture, imply strong benefits to simultaneous observation of the entire data cube, resulting in a homogenous data set with well-behaved noise characteristics (low levels of systematic effects).

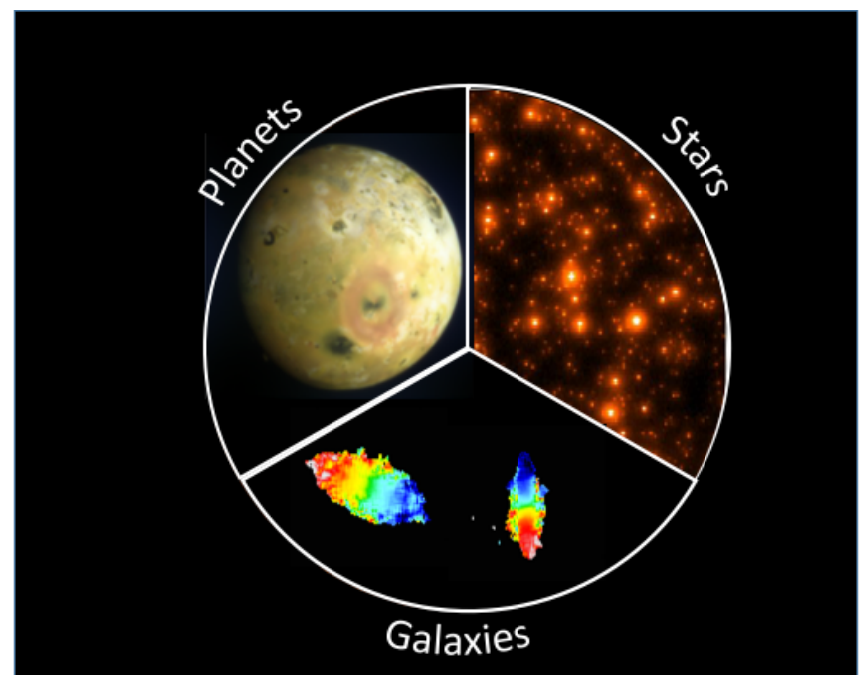

Figure 1: Illustration of simulated observations made with HARMONI in the three key areas of E-ELT science drivers: stars, planets and galaxies. The images show Io, a star field in a local group galaxy, and emission line kinematics of high redshift galaxies (as observed using the $\mathrm{H} \alpha$ line at redshifts of 2-3).

The scientific need for a single field integral field spectrograph for the European Extremely Large Telescope (E-ELT) was set out in the Top Level Requirements document from the European Southern Observatory's Project Science Team (ESO PST)[4]. This followed the decision by ESO to include a visible and near-infrared integral field spectrograph (ELT-IFU) in the first light instrument complement for the E-ELT. The details of the ESO E-ELT instrument roadmap are presented in [5]. HARMONI, the instrument that fulfills the requirements set for ELT-IFU, is foreseen to be a workhorse instrument that enables a wide variety of observing programmes, including those areas that form the three pillars of the E-ELT science case, as shown in Figure 1.

HARMONI provides a range of spaxel scales and spectral resolving powers, which permit the user to optimally configure the instrument for a wide range of science programmes; from ultra-sensitive to diffraction limited spatially resolved physical (via morphology), chemical (via abundances and line ratios) and kinematic (via line-of-sight velocities) studies of astrophysical sources. Specifically, it will provide medium resolution, $R(\equiv \lambda / \Delta \lambda)$ of $\sim 3500, \sim 7500$ and $\sim 20000$ spectroscopy at spatial resolutions ranging from seeing limited to diffraction limited, across the visible and near-infrared wavelength range ( 0.5 to $2.4 \mu \mathrm{m})$.

\section{TECHNICAL SPECIFICATIONS}

As part of the work leading up to the start of Phase B (Preliminary Design Phase), ESO and the HARMONI consortium jointly agreed on the technical specifications for HARMONI and its associated natural guide star adaptive optics (SCAO) wavefront sensing (WFS) system. The laser tomographic adaptive optics (LTAO) WFS system was not sufficiently well defined to allow technical specifications to be set, instead, it was agreed that these would be developed during the Phase B study of the LTAO WFS system. 
The technical specifications were further refined in a cost v/s capability trade-off process that took place in early 2015 . The goal of the trade-off exercise was to provide the most versatile instrument with the best performance, whilst staying within the available hardware cost budget and staff effort. The low resolution mode (using a prism as the disperser) providing simultaneous visible and near-infrared coverage at $\mathrm{R} \approx 500$, was omitted from the technical specifications, following a prioritization performed by the ESO Project Science Team (PST) [4]. We present below the key functional and performance requirements, reproduced from the technical specifications document.

\subsection{Assumptions}

The technical specifications for the HARMONI IFS assume a $39 \mathrm{~m}$ E-ELT, with mirrors M4 and M5 capable of delivering adaptive optics correction for atmospheric turbulence and wind-shake. A detailed description of the latest update to the E-ELT baseline design can be found in Tamai et al. [6]. We also assume a 6 laser guide star (LGS) LTAO system. HARMONI is assumed to have $84 \mathrm{~K} \times 4 \mathrm{~K}$ near-IR detectors, with specifications similar to the H4RG detectors manufactured by Teledyne Inc. [8].

\subsection{Wavefront Control Modes}

HARMONI will operate in three modes:

- No AO - no adaptive optics correction of atmospheric turbulence is envisaged in this mode. However, the EELT's Central Control System (CCS) provides correction of wind-shake, flexure and other perturbations, so as to achieve "seeing-limited" performance.

- $\quad \mathrm{SCAO}$ - a bright natural guide star (NGS) is used to provide the reference for adaptive optics correction of atmospheric turbulence. The instrument participates in wavefront control by supplying NGS wavefront sensor information to the real time computer (RTC).

- LTAO - six laser guide stars, and one or more natural stars will be used to sense the atmospheric turbulence. The HARMONI LTAO system shall participate in wavefront control by providing all the sensor information to the real time computer. The E-ELT's M4 and M5 are foreseen to be the only deformable components.

\subsection{Fields of View and Spatial element scales}

HARMONI's IFS has four spaxel scales in its baseline design, so as to optimally match the sensitivity and spatial resolution to the observing programme. Of these, the coarsest spaxel scale is set by the maximum étendue that can be provided by the spectrograph cameras whilst ensuring adequate image quality over the entire detector area, assuming 15 $\mu \mathrm{m}$ pixel size at the detector. At the other extreme, the finest spaxel scale is matched to the diffraction limited core of the PSF delivered by the telescope. The specifications are intended to fulfill the scientific requirements without curbing design freedom. Thus, they do not specify exact numbers for each spaxel scale, or fields of view.

HARMONI will have a rectangular field of view on the sky, with an aspect ratio not exceeding 2:1. The instrument will provide at least three spatial resolution scales in all wavelength settings. The aspect ratio of each spatial element, as projected on the sky, shall not exceed 3:1. The finest spatial scale in the near-infrared shall at least Nyquist sample the full width at half maximum of the best point spread function provided by the (adaptive) telescope at $1.45 \mu \mathrm{m}$. The coarsest near-infrared spaxel scale shall have an area of at least 1250 mas $^{2}(25 \times 50$ mas). By contrast, the coarsest visible spaxel scale shall have an area of at least 5000 mas $^{2}(100 \times 50$ mas $)$.

The field of view in the near infrared at the coarsest scale shall be at least $38 \operatorname{arcsec}^{2}$, and for the finest scale at least 0.5 $\operatorname{arcsec}^{2}$. At visible wavelengths, the field of view at the coarsest spaxel scale shall be at least $9 \operatorname{arcsec}^{2}$. Furthermore, the fields of view at all spatial scales shall lie within the largest near-infrared field of view.

\subsection{Wavelength coverage and resolving power}

HARMONI is a visible and near-infrared integral field spectrograph, specified to cover the wavelength range $0.5-2.4$ $\mu \mathrm{m}$ (with a goal of 0.43 to $2.45 \mu \mathrm{m}$ ). Three different resolving powers are foreseen, $R(\equiv \lambda / \Delta \lambda)$ of $\sim 3500, \sim 7500$ and $\sim 20000$. As each grating provides a range of resolving powers (to first order $\Delta \lambda$ stays fixed for a slit-width limited grating spectrograph), the specification requires a minimum resolving power at the central wavelength for each disperser. The cut-on and cut-off wavelengths of the various settings are set to match the atmospheric transmission windows in the near-infrared, wherever appropriate. The various settings and resolving powers are defined as follows: 
Table 1: list of HARMONI spectral resolving powers and corresponding instantaneous wavelength coverage.

\begin{tabular}{|c|c|c|c|}
\hline Name & $\mathbf{R}_{\min }(\equiv \lambda / \Delta \lambda)$ & $\lambda_{\min }(\mu \mathbf{m})$ & $\lambda_{\max }(\mu \mathbf{m})$ \\
\hline $\mathrm{V}+\mathrm{R}$ & 3000 & $0.5($ goal 0.43$)$ & 0.8 \\
\hline $\mathrm{IzJ}$ & 3000 & 0.83 & 1.35 \\
\hline $\mathrm{HK}$ & 3000 & 1.45 & $2.4($ goal 2.45$)$ \\
\hline $\mathrm{Iz}$ & 7000 & 0.83 & 1.05 \\
\hline $\mathrm{J}$ & 7000 & 1.05 & 1.32 \\
\hline $\mathrm{H}$ & 7000 & 1.45 & 1.8 \\
\hline $\mathrm{K}$ & 7000 & 1.97 & 2.40 \\
\hline $\mathrm{J}_{\text {high }}$ & $20000 \pm 2000$ & $1.19(\mathrm{TBC})$ & $1.30(\mathrm{TBC})$ \\
\hline $\mathrm{H}_{\text {high }}$ & $20000 \pm 2000$ & $1.54(\mathrm{TBC})$ & $1.67(\mathrm{TBC})$ \\
\hline $\mathrm{K}_{\text {high }}$ & $20000 \pm 2000$ & $2.10(\mathrm{TBC})$ & $2.28(\mathrm{TBC})$ \\
\hline
\end{tabular}

An additional fourth slot for a high resolution grating is planned in the IFS baseline design, the wavelength range will be specified by the end of the Preliminary Design Phase. The spectral sampling is specified to be at least two detector pixels per resolution element for all wavelength settings.

\subsection{Differential atmospheric refraction}

HARMONI is not foreseen to have an atmospheric dispersion corrector, as preliminary analysis showed that differential atmospheric diffraction is unlikely to have a significant impact for most observations. Individual wavelength channels of the final data cube can always be shifted relative to each other to eliminate the impact of atmospheric dispersion. However, this does impact the field of view common to all wavelengths within the data cube, an effect that must be taken into account. In addition, as the atmospheric dispersion varies during a long exposure, it can only be fully compensated at a single (guide) wavelength. All other wavelength channels will see a slightly elongated long exposure PSF. Another effect, apparent in long exposures, is the smearing of the elongated PSF due to changes in the parallactic angle during the exposure. Unfortunately, these secondary effects cannot be corrected by post-processing, and could limit the final image quality for some observations. However, reducing the exposure length allows these effects to be mitigated, with only a modest impact on performance.

\subsection{Image and spectrum quality}

The image quality of an image slicer based integral field spectrograph is difficult to quantify, as the opto-mechanics located past the image slicer only impact the spatial image quality in only one dimension (but do impact the spectral line spread function as well). Diffraction effects are important when considering spaxel scales close to, or smaller than the telescope's diffraction limit. Anamorphic magnification of the sky image at some spaxel scales, introduced to achieve square spaxels as projected on the sky, also have an impact. Testability of any image quality requirement in the laboratory is also an important consideration. For an integral field spectrograph, it may be argued that the relevant requirement should be phrased in terms of ensquared energy within a specific box and spectral resolution, rather than in terms of image spread (full width at half maximum, or FWHM) or Strehl ratio. The latter can be particularly difficult to measure in the reconstructed image of an integral field spectrograph, given the small field of view at small spaxel scales.

HARMONI's image quality is specified in terms of ensquared energy for the two intermediate spaxel scales (nominally foreseen to be $20 \times 20$ mas, and $10 \times 10$ mas). For these two scales, the instrument's long exposure PSF shall have an ensquared energy $\geq 75 \%$ in a $2 \times 2$ spaxel box. For the coarsest and finest spaxel scales, the image quality is specified in terms of FWHM, as spatial resolution, rather than sensitivity alone, is deemed important in those two scales. At the coarsest spaxel scale (foreseen to be used for seeing limited observations), the specifications require that the instrument not degrade the FWHM of a point source image delivered by the telescope by more than $10 \%$. At the finest spaxel scale, 
the instrument shall not degrade by more than $20 \%$ the FWHM of a Nyquist sampled Gaussian input PSF. For all spaxel scales, the specification applies to a single exposure, and excludes effects due to interpolation on to a regular grid.

For all spaxel scales, the spectrograph is constrained to not degrade by more than $10 \%$ (TBC) the nominal spectral resolving power of the disperser for all wavelengths (i.e. allow for the natural variation of resolving power with wavelength intrinsic to the grating). The anamorphic magnification introduced by the disperser does impact the slit width, and therefore the spectral resolving power of a slit width limited spectrograph. This can be traded-off against the requirement to at least Nyquist sample the spectral resolution element at all wavelengths, so as to meet the specifications. In addition, the monochromatic FWHM of any spectral line shall vary by less than $10 \%$ across all spaxels.

\subsection{Throughput, scattered light, instrument background and ghosts}

The instrument transmission (excluding the detectors) is specified to be at least $30 \%$, averaged over the field of view and the central $80 \%$ of the instantaneous wavelength range. Given the complex shape of the E-ELT pupil, and some unavoidable pupil wander (the interface control document (ICD) specifies a pupil wander of $\pm 0.5 \%$ ), we found that placing a cold pupil mask matched to the pupil shape did not provide a significant benefit. Thus, the throughput specification is defined for the largest circular pupil (inner and outer edges) that fits within the E-ELT primary, the socalled "all glass" area.

The total additional background introduced by the instrument, relative to that from the sky and telescope combined, is specified to be no more than $20 \%$. The excess background is the median value measured over all detector pixels, so as to allow for wings of night sky emission lines caused by the disperser and other sources of scattering within the instrument. The scattered light level and the thermal background introduced by the instrument itself are not specified separately, as only the total excess background is relevant to the performance. An overall specification allows for some design freedom and trade-offs.

Ghost images generated within the instrument are limited to be no brighter than 5 parts in 10000 at their peak intensity.

\subsection{SCAO and high contrast}

HARMONI will include a wave front sensor for full atmospheric turbulence correction using bright natural guide stars (SCAO WFS). As the brightness of the reference star required is independent of telescope size (only depending on the size of each sub-aperture), the expected sky coverage is tiny, of order $1 \%$. However, the SCAO mode is vital to achieving the scientific potential of HARMONI as it offers the simplest path to achieve diffraction limited performance with the E-ELT. Consequently, providing SCAO WFS capability is a key requirement for HARMONI.

As adaptive correction of the incoming wavefront is provided by the M4 deformable mirror and the M5 tip-tilt mirror of the E-ELT, the instrument requirements are limited to providing wave front sensing for SCAO. The technical specification requires the SCAO WFS to sense the residual wavefront error (when operating in closed loop) to an accuracy of better than $100 \mathrm{~nm}$ rms, when guiding on a star with $\mathrm{M}_{\mathrm{V}}<12$ (spectral type G0) under median seeing conditions, and with a latency of less than $1.5 \mathrm{~ms}$ when operating at $500 \mathrm{~Hz}$.

In addition, the SCAO WFS should be able to acquire a natural guide star within 15" radius of the field centre. The patrol field is chosen based on the cross-over point for expected performance of the SCAO and LTAO systems. Solar system objects, such as the Jovian and Saturnian satellites, are potential targets for HARMONI observations. The SCAO system is required to be able to guide using extended sources up to $2.5 \mathrm{arcsec}$ in diameter (goal $4 \mathrm{arcsec}$, the size of Uranus) to enable these observations.

High contrast observing, such as spectroscopy of directly imaged extra solar planets, is one of the key drivers for the EELT. Every effort has been made to include constraints arising from the needs of high contrast observations in the design of HARMONI. However, given the uncertainty of the interface, and inadequate knowledge of the detailed design and implementation of some of the E-ELT's functions, it was not possible to specify the contrast achievable by HARMONI (including its SCAO WFS). Instead, we proposed a goal of 1 part per million of contrast, at a distance of 200 mas from a guide star brighter than $\mathrm{M}_{\mathrm{V}}=9$, in the best quartile seeing conditions. This goal applies to the final reduced data cube, including differential techniques such as angular differential imaging and post-processing such as spectral deconvolution. 


\section{BASELINE ARCHITECTURE \& TRADE-OFFS}

There are only small modifications to the integral field spectrograph conceptual design developed during the Interim Study Phase, mostly as a result of cost v/s capability trade-offs. However, incorporating the HARMONI Laser Tomographic Adaptive Optics (LTAO) system as part of the instrument has led to substantial changes to the instrument architecture and layout, which we discuss in the next few sub-sections.

\subsection{Optimal LGS Asterism diameter}

Early concepts for implementing LTAO at the E-ELT investigated a variety of options for separating the sodium laser beacon light at $589 \mathrm{~nm}$ from the science light path, as well as providing natural guide star (NGS) low order wave-front sensing (WFS) for correcting tip-tilt (and slow focus) aberrations. The low order NGS WFS use an annular "technical field" centered on the IFS field of view. The large defocus of the laser guide stars (LGS) (several meters for $90 \mathrm{~km}$ LGS altitude) added further difficulty, as did the HARMONI wavelength range that encompassed wavelengths on either side of the sodium laser beacon wavelength.

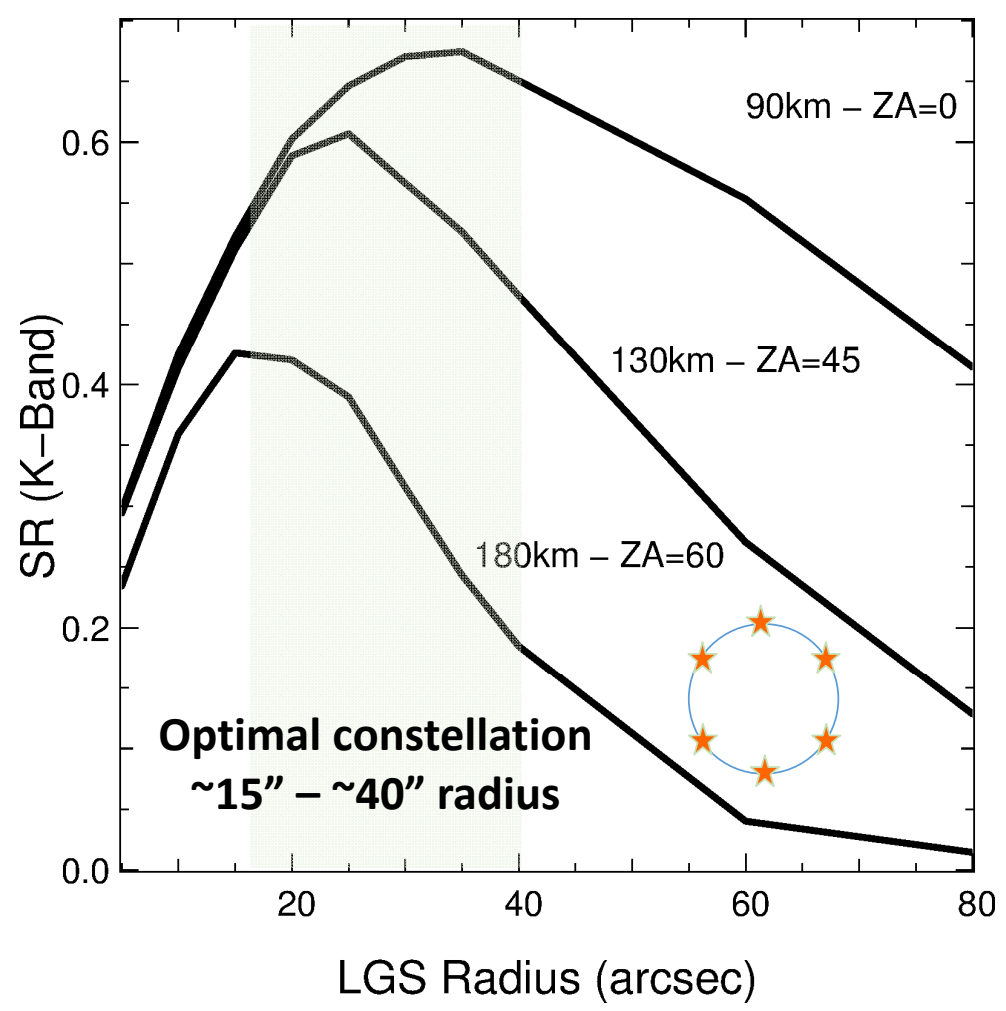

Figure 2:Expected variation of performance (Strehl ratio) with LGS asterism diameter for the HARMONI LTAO system.

The lines represent different zenith angles for the observations.

New simulations were carried out to explore the dependence of LTAO system performance on laser star asterism diameter, always assuming 6 equi-spaced laser guide stars on a ring. Figure 2 shows the results of these simulations. As expected, optimum performance for a given LGS altitude is obtained when the laser stars lie close to the rim of the cylinder formed by projecting the telescope pupil along the telescope optical axis. Furthermore, observations at angles away from zenith imply more distant laser guide stars, and additionally lead to a $\cos (\mathrm{ZD})$ foreshortening effect, which further reduces the optimal asterism diameter.

At Phase A, and during the interim study phase [7], simulations appeared to indicate that increasing the size of the laser asterism to more than 1 arc minute radius would have a small impact on performance. On the other hand, it would support a central "hole" free of laser light for a small $\left(10^{\prime \prime}\right.$ to $\left.30^{\prime \prime}\right)$ science field. However, newer simulations showed a steep drop in performance for large asterism diameters, and this has been independently verified using detailed end-to- 
end simulations. The performance gain from using a small asterism diameter, at or close to the optimum value, more than compensates for the potential throughput loss incurred by inserting a dichroic in the beam path. Consequently, a key decision at the start of the preliminary design was to adopt an LGS asterism diameter of 1 arc minute or less, and to use a dichroic to separate the laser beacon light from the science and NGS light paths. Furthermore, it was decided to incorporate a variable asterism diameter in the LGS WFS design, so that the optimal LGS asterism could be maintained for all zenith distances.

\subsection{Gravity invariant cryostat}

Throughout the Phase A study, and the interim study phase, we opted either for a fixed cryostat geometry, or for a gravity invariant cryostat, rotating about a vertical axis to compensate for field rotation. The primary driver was the stability of the calibrations, particularly the wavelength calibration at high spectral resolving power. Given the rapid and strong variation of the night sky emission lines at near-infrared wavelengths, successful sky subtraction to a high degree of accuracy relies on the stability of the wavelength calibration. This is even more critical for integral field spectrographs, where the spectra tend to be just Nyquist sampled. Other instrument designs for extremely large telescopes [1],[2] have also adopted the same approach.

For the HARMONI design, the trade-off study showed a strong preference for a gravity invariant cryostat rotating about a vertical axis over a fixed cryostat with an optical de-rotator. To achieve adequate sky coverage ( $>50 \%$ on average), the required field of view for natural guide stars to sense the tip-tilt (and focus) terms of atmospheric turbulence and residual wind-shake during LTAO operation is 2 arcmin in diameter [3]. Given the large plate scales at the E-ELT focus, it is prohibitive to build an optical de-rotator (K-mirror) for such a large field of view. Mounting the LTAO NGS tip-tilt wave front sensors on top of a cryostat rotating about a vertical axis provides two key advantages: the differential motion (noncommon path) between the tip-tilt sensors and the IFS focal plane is reduced to a minimum, and the vertical rotation axis ensures that the NGS sensors themselves are mounted in a gravity invariant manner.

An alternative, side-looking fixed cryostat with an optical de-rotator for the science field would have reduced the need for a (cold) focal plane relay, but would need a horizontal de-rotation axis for the NGS probes, which would be subjected to varying gravity vector. Furthermore, since the field de-rotation for the IFS and the NGS probes was not directly coupled, it could lead to differential errors. Also, given the $1 \mathrm{~m}$ back focal distance of the E-ELT, it would not be possible to separate the LGS light from the natural star light.

\subsection{Cold focal plane relay}

The trade-offs described in the previous section suggested that best performance would be obtained for a gravity invariant cryostat rotating about a vertical axis to compensate for field de-rotation, with the natural star sensors corotating with the IFS cryostat. The field of view required by the LTAO NGS tip-tilt probes is also sufficient for the SCAO wavefront sensor, and the secondary guiding sensor (required for controlling motion \& rotation relative to the PSF at $\sim 1 \mathrm{~Hz}$ frequencies for seeing limited operation), so they are located in the same co-rotating volume. As the telescope back focal distance is only $1000 \mathrm{~mm}$, there is not enough path length available to simply fold the telescope light path into the instrument. Instead a full focal plane relay is required that provides adequate image quality (close to diffraction limited) across a 2 arcminute diameter field of view. Six different options for the focal plane relay were considered in the trade-off study, showing a clear preference for a modified Offner based relay system.

Excess thermal background from room temperature optical components strongly limits the sensitivity of HARMONI at wavelengths longer than $1.6 \mu \mathrm{m}$. As both LTAO and SCAO performance improves rapidly with increasing wavelength, limitations arising from excess thermal background would strongly compromise the science goals of HARMONI. A detailed analysis was carried out to quantify the effect of increased emissivity on performance at $\mathrm{K}$ band. It showed that $30 \%$ increase in emissivity (relative to that from the telescope + sky) led to 0.15 mag decrease in limiting magnitude in a 5 hour long exposure, or required $15 \%$ longer exposure time to compensate for the excess thermal background noise. The telescope has 7 warm reflections, and an expected emissivity of $20.5 \%$, so at most 3 warm surfaces could be tolerated if the instrument is to introduce no more than $20 \%$ of the thermal background from the telescope + sky. The laser light dichroic and the cryostat window already use up most, if not all, of the available budget, so it is necessary to cool the relay optics to $25-30^{\circ} \mathrm{C}$ below the ambient to keep the excess thermal background within acceptable limits. Cooling the optics below the dew point necessitates the need for a window. However, introducing a dry gas seal on the exit side of the relay serves the dual purpose of removing the need for an exit window, and of reducing the cryostat window temperature so that it no longer contributes to the thermal emissivity budget. 


\subsection{Baseline architecture}

The system configuration adopted as per the baseline architecture is shown in Figure 3 below. The entire instrument, including its associated adaptive optics sensing systems (for SCAO and LTAO) operate in three temperature zones: ambient, cold and cryogenic. The calibration unit, the laser dichroic and its exchange mechanism, and the LGS wavefront sensor operate at ambient temperature. The focal plane relay operates at cold temperatures (approximately $-30^{\circ} \mathrm{C}$ ), so as the minimize the excess thermal background during $\mathrm{K}$ band operations. The cold environment is shared by all the natural star sensing systems (LTAO-NGS, SCAO and secondary guiding). Systems inside the cryostat, which include the integral field spectrograph and its pre-optics system, operate at cryogenic temperatures $(\sim 130 \mathrm{~K})$. All elements are gravity invariant. The cryostat de-rotates to ensure a fixed field orientation on the sky, all natural star sensors co-rotate with it. The LGS wave front sensing system de-rotates with the telescope pupil so as to keep the LGS asterism geometry fixed. The focal plane relay, the LGS dichroic and its exchange mechanism, and the calibration module are fixed w.r.t. the Nasmyth platform and the pre-focal station (PFS).

LTAO operation: During LTAO operation, the LGS dichroic is inserted into the telescope beam path, reflecting all light shortward of $590 \mathrm{~nm}$ to the LGS WFS system. Given the large size of the LGS dichroic, we expect that good transmission ( $>90 \%)$ is possible only beyond $630 \mathrm{~nm}$. The telescope focal plane is re-imaged by the focal plane relay to the top of the cryostat for all wavelengths in the $630-2450 \mathrm{~nm}$ range. The layout of the scientific and technical fields in the relayed natural star focus is illustrated in Figure 4. The science light passes through the cryostat window into the IFS cryostat. The LTAO NGS wavefront sensors (for low order correction, namely tip, tilt and focus) patrol the 2 arcmin diameter technical field to choose suitably bright stars. The secondary guiding sensor provides information about the absolute pointing (world coordinate reference frame).
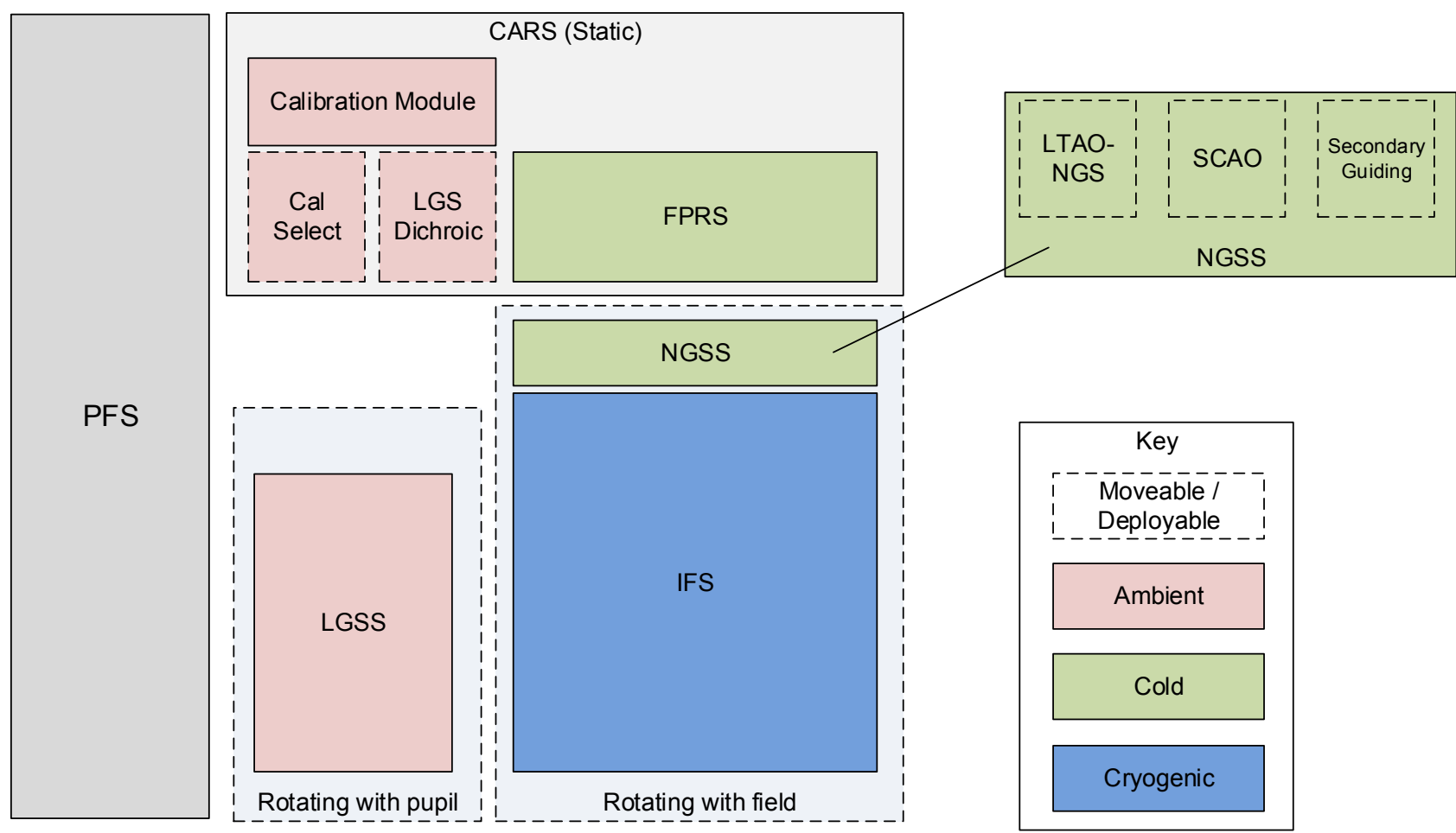

Figure 3:Top level block diagram of HARMONI, including the H-LTAO system. Also shown are the thermal environments of each component / block.

SCAO operation: During SCAO operation, the LTAO dichroic is retracted, and light of all wavelengths (450 nm -2450 $\mathrm{nm}$ ) is relayed by the focal plane relay to the top of the HARMONI cryostat. A SCAO dichroic located in the science light path just before the relayed focus redirects light short-ward of $800 \mathrm{~nm}$ (a second option of $1000 \mathrm{~nm}$ is being 
explored) to the SCAO wavefront sensor. The SCAO sensor can pick any bright star within a 15 arc sec radius patrol field centered on the science field centre. The LTAO and secondary guiding sensors are not used during SCAO operation.

Seeing limited operation: When operating without adaptive optics correction of atmospheric turbulence (wind shake, vibrations and differential motions still need to be corrected), the LTAO dichroic is retracted, and light of all wavelengths is relayed to the IFS. The secondary guiding sensor patrols the 2 arcmin technical field, and uses a faint offaxis natural star to compensate for slowly evolving errors in position and focus. The LTAO and SCAO sensors are not used during seeing limited operation.

Calibration: The calibration fold mirror can be inserted in the light path, blocking the light from the telescope, and allowing light from the calibration unit to be fed to various sub-systems of HARMONI. The calibration unit provides wavelength calibration, flat field and point-like sources for calibrating the IFS. In addition, it also provides the ability to calibrate the SCAO wave front sensor, the LTAO natural star low order wavefront sensors, and the secondary guiding sensor.

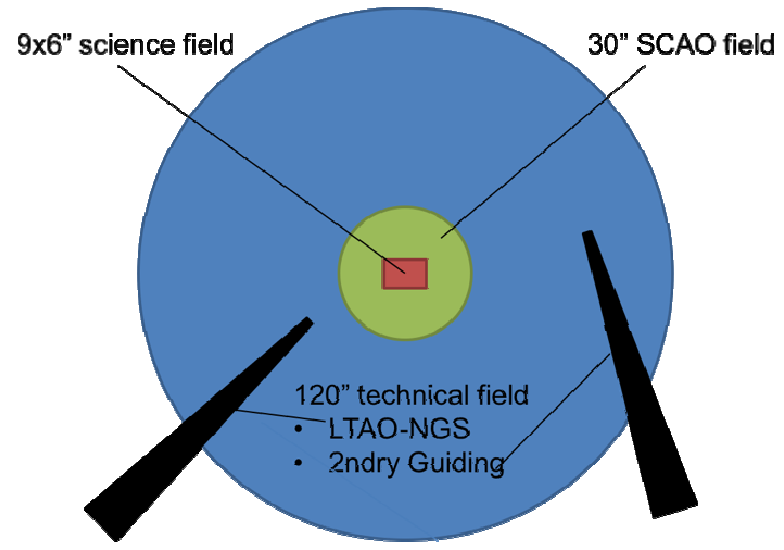

Figure 4:Layout of the scientific and technical fields in the relayed natural star focal plane at the top of the HARMONI cryostat. The science field of view is shown in red, the SCAO reference star patrol area in green, and the LTAO NGS (and secondary guiding) patrol area in blue.

The HARMONI cryostat houses several sub-systems. The pre-optics system provides scale-changing optics to permit choice of one of four spaxel scales. It also incorporates the fast shutter, the band pass filters (for order sorting of the dispersers), the focal plane mask wheel carrying field stops and calibration masks, the cold pupil stop and pupil imaging optics for verifying the alignment of the instrument pupil with the telescope pupil. The IFU sub-system provides field splitting and image slicing, as well as anamorphic magnification. It relays the input field into 4 long slits that feed the light to the spectrograph modules.

Four spectrograph modules, each incorporating 10 near-infrared dispersers (and two incorporating visible dispersers as well) produce spectra of the input slits on to two $15 \mu \mathrm{m}$ pixel $4 \mathrm{~K} \times 4 \mathrm{~K}$ near-infrared detectors located side-by-side. The spectral length is $4 \mathrm{~K}$ pixels. The detectors operate at a well controlled temperature lower than that of the cryostat to ensure good cosmetics, low dark current, and stable performance. A detailed description of the IFS sub-systems, the design of which hasn't evolved significantly since the interim study phase, is presented in Thatte et al. [7].

\subsection{Nasmyth platform volume allocation and layout}

HARMONI will be located at one of the side-looking ports at the E-ELT Nasmyth focus. The instrument volume, as defined in the interface control document (ICD) is $5000 \mathrm{~mm}$ wide (centered on the telescope optical axis), $8750 \mathrm{~mm}$ long and $10000 \mathrm{~mm}$ tall. The telescope focus is located $1000 \mathrm{~mm}$ inside the instrument volume (along the length dimension), at a height of $6000 \mathrm{~mm}$.

Figure 5 shows a CAD rendering of the instrument located at the E-ELT Nasmyth platform. The telescope focus is located inside the olive green box, which represents the cold box that houses the focal plane relay. The cold box is maintained at $25-30^{\circ} \mathrm{C}$ below ambient, to suppress excess thermal background from warm optical components. It will be filled with dry nitrogen gas to avoid condensation of ambient water vapour on opto-mechanics and electronic 
components. A rotating gas seal at the exit side maintains the slight overpressure of dry nitrogen within the relay cold box. As the relay is fixed, it is held on stiff, earthquake-proof, supports that surround the rotating cryostat structure. The top of the cryostat is domed, allowing for a thinner outer shell without compromising vacuum integrity and ensuring a stable mating surface for the wavefront sensor module. This houses the LTAO NGS, the SCAO and the secondary guiding sensors, all located within the same cold environment as the focal plane relay. The entire cryostat and wavefront sensor assembly is located on a de-rotator that allows for observing in either "field fixed" or "pupil fixed" configurations (the latter is used for angular differential imaging for high contrast SCAO observations). The outside of the cryostat houses electronics racks containing drive and control electronics for the detectors and the moving mechanisms..

The green box houses the LTAO dichroic (retractable), the calibration unit and its retractable fold mirror. When the LTAO dichroic is inserted in the beam path, it sends the sodium laser light to the LGS wave front sensor module (yellow vertical cylinder) that houses six laser guide wavefront sensors and their focus compensation system. The entire LGS WFS module is mounted on a separate de-rotator that allow the LGS WFS to maintain a "pupil fixed" geometry that tracks the laser star asterism on the sky.

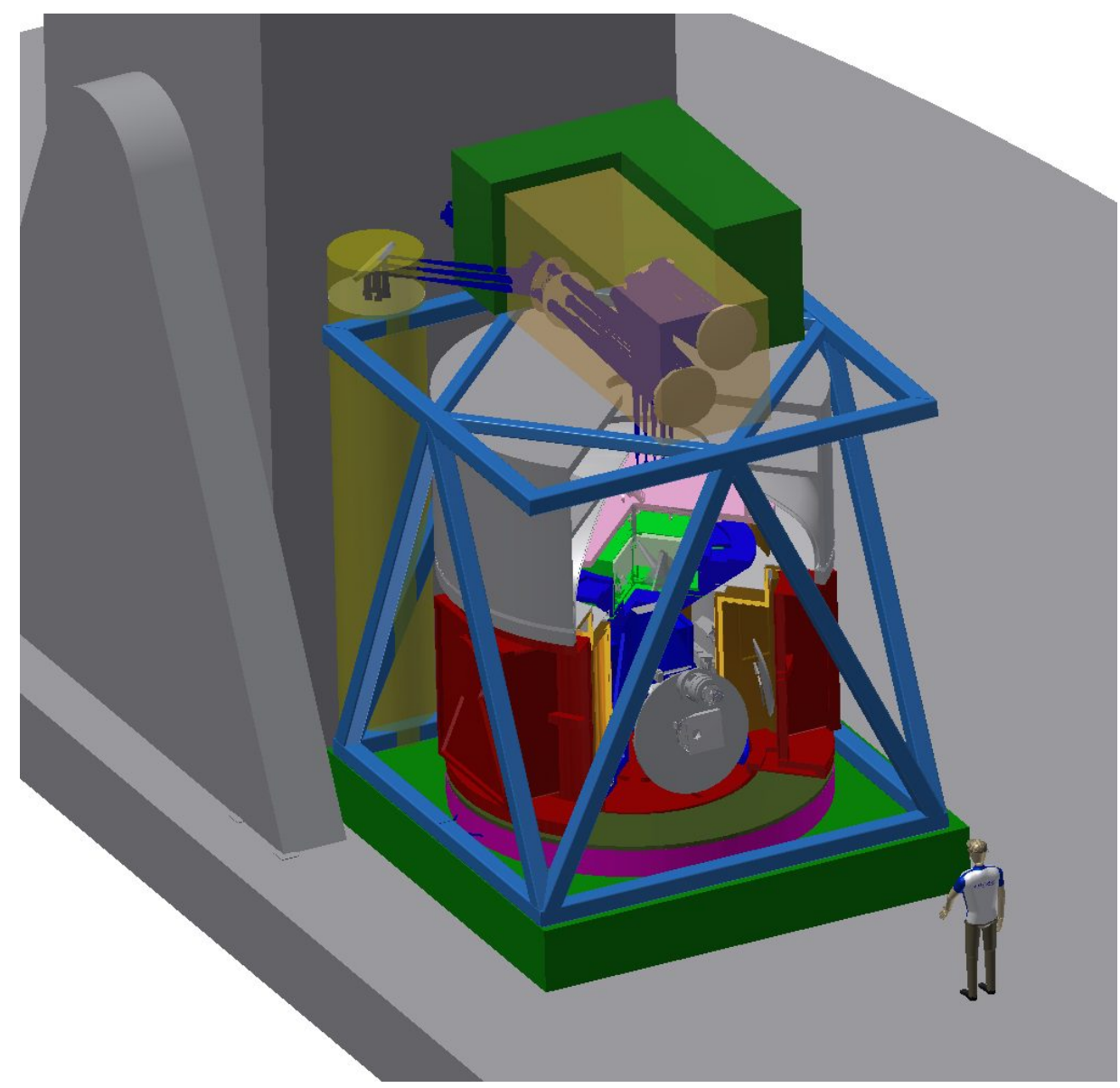

Figure 5: CAD rendering of the various sub-assemblies of HARMONI mounted on the Nasmyth platform (light grey structure at bottom). The telescope pre-focal station (PFS) is shown in dark grey, the HARMONI IFS cryostat in red, the rotator in pink, the wavefront sensor modules on top of the cryostat in light grey, the calibration module \& dichroic exchange unit in dark green, the LGS WFS module (vertical cylinder) in yellow, the focal plane relay unit in olive green, and the support structure for it in blue. 


\section{PERFORMANCE PREDICTIONS}

Using the instrument configuration as defined by the baseline architecture, and with simulated PSFs computed using the latest LGS asterism diameters, we have estimated the sensitivity of the instrument in its various configurations (combination of spaxel scale and spectral resolution). We made reasonable assumptions for the atmospheric conditions, AO performance, instrument throughput, detector noise and efficiency, etc. We assumed median seeing conditions as specified in the 35 layer ESO model for Cerro Armazones atmospheric turbulence, a night sky background level commensurate with observing between the $\mathrm{OH}$ lines, the E-ELT "all glass" aperture area (minus the obscuration by the secondary mirror supports, or "spiders"). The telescope throughput is specified by the interface control document (ICD) between the E-ELT and its instruments. Observations were assumed to be $30^{\circ}$ from zenith. The instrument throughput is taken to be $30 \%$ on average, including the focal plane relay, the near-IR detectors are assumed to have multiple-sample read noise of 2.8 electrons, a dark current of $0.0053 \mathrm{e} / / \mathrm{s} / \mathrm{pix}$, and QE of $95 \%$. The visible detectors are assumed to have a read noise of 2 electrons, and a dark current of $2 \mathrm{e}^{-} / \mathrm{hr} / \mathrm{pix}$. The table below shows the limiting magnitude (in $\mathrm{AB}$ mag units) of a point source, for which HARMONI at the E-ELT can expect to achieve a SNR of 5 in the continuum, in 5 hours ( $20 \times 900$ second exposures) of on-source integration time under the conditions listed above, assisted by LTAO, in the $\mathrm{R}$ and $\mathrm{H}$ bands. The SNR is for an extracted spectrum using a $2 \times 2$ spaxel box centred on the object. Sky subtraction is assumed to have no impact on on-source exposure time, only the detector and background noise is increased.

Table 2: HARMONI limiting magnitudes $(\mathrm{AB})$ for SNR of 5 in 5 hours on-source.

\begin{tabular}{|l|c|c|c|c|c|c|c|c|}
\hline \multirow{2}{*}{$\begin{array}{l}\text { Spectral } \\
\text { Resolution }\end{array}$} & \multicolumn{2}{|c|}{4 mas } & \multicolumn{2}{c|}{10 mas } & \multicolumn{2}{c|}{20 mas } & \multicolumn{2}{c|}{$30 \times 60$ mas } \\
\cline { 2 - 9 } & $R_{A B}$ & $H_{A B}$ & $R_{A B}$ & $H_{A B}$ & $R_{A B}$ & $H_{A B}$ & $R_{A B}$ & $H_{A B}$ \\
\hline & \multicolumn{8}{c|}{ Point source (AB mag) } \\
\hline 3500 & 23.97 & 26.06 & 24.83 & 27.05 & 25.11 & 27.26 & 25.74 & 26.76 \\
\hline 7500 & & 25.23 & & 26.24 & & 26.50 & & 26.11 \\
\hline 20000 & & 24.17 & & 25.19 & & 25.47 & & 25.17 \\
\hline
\end{tabular}

The table clearly shows that optimal point source sensitivity is achieved for the 20 mas spaxel scale, as also computed by Zieleniewski et al. [9]. Our numbers differ from the values computed by Zieleniewski et al. [9] due to improved ensquared energies arising from the reduced (optimal) LGS asterism diameters.

\section{REFERENCES}

[1] Davies, R. I., et al., "MICADO: first light imager for the E-ELT," Proc. SPIE 9908, 9908-73 (2016).

[2] Larkin, J., et al., "The Infrared Imaging Spectrograph (IRIS) for TMT: instrument overview," Proc. SPIE 9908, 9908-70 (2016).

[3] Neichel, B., et al., "The HARMONI laser tomography module," Proc. SPIE 9909, 9909-9 (2016)

[4] Padovani, P., "Top level requirements for ELT-IFU" ESO science web site, $<$ http://www.eso.org/sci/facilities/eelt/docs/ESO-191883_2_Top_Level_Requirements_for_ELT-IFU.pdf $>$ (23 June 2015)

[5] Ramsay, S. K., et al., "Progress along the E-ELT instrumentation roadmap," Proc. SPIE 9908, 9908-67 (2016)

[6] Tamai, R., et al., "The E-ELT Program status," Proc. SPIE 9906, 9906-31 (2016).

[7] Thatte, N. A., et al., "HARMONI: the first light integral field spectrograph for the E-ELT," Proc. SPIE 9147, 914725 (2014).

[8] Zandian, M., et al., "Performance of science-grade HgCdTe H4RG-15 image sensors," Proc. SPIE 9915, 991513 (2016)

[9] Zieleniewski, S., et al., "HSIM: a simulation pipeline for the HARMONI integral field spectrograph on the European ELT, " MNRAS 453, 3754Z (2015). 